\title{
Microscopic conditions favoring itinerant ferromagnetism: Hund's rule coupling and orbital degeneracy
}

\author{
K. Held and D. Vollhardt \\ Theoretische Physik III, Elektronische Korrelationen und Magnetismus, Universität Augsburg, D-86135 Augsburg, Germany
}

(July 10, 2021)

The importance of Hund's rule coupling for the stabilization of itinerant ferromagnetism is investigated within a twoband Hubbard model. The magnetic phase diagram is calculated by finite-temperature quantum Monte Carlo simulations within the dynamical mean-field theory. Ferromagnetism is found in a broad range of electron fillings whereas antiferromagnetism exists only near half filling. The possibility of orbital ordering at quarter filling is also analyzed.

\section{INTRODUCTION}

Investigations of the microscopic mechanisms responsible for the stability of metallic ferromagnetism have recently received renewed attention. In particular, it was finally established that itinerant ferromagnetism is indeed stable in the one-band Hubbard model at intermediate on-site interactions, an important condition being a properly tuned kinetic energy with a pronounced peak in the density of states (DOS) near the band edge 1 . 9. For real ferromagnets, e.g., the transition metals $\mathrm{Fe}$, $\mathrm{Co}$, and $\mathrm{Ni}$, quite a different microscopic mechanism for ferromagnetism, based on Hund's rule coupling in the presence of orbital degeneracy, is also expected to be relevant. It was first suggested by Slater 10] and then stressed by van Vleck 11] that the intra-atomic exchange leading to "Hund's rule atomic magnetism" might be responsible for bulk ferromagnetism, i.e., the hopping of electrons or holes might lead to a bulk ordering of preformed atomic moments.

Concrete calculations based on microscopic models began with Roth 12 who considered a two-band extension of the Hubbard model

$$
\begin{aligned}
\hat{H}= & -t \sum_{i j \nu \sigma} \hat{c}_{i \nu \sigma}^{\dagger} \hat{c}_{j \nu \sigma}+U \sum_{i \nu} \hat{n}_{i \nu \uparrow} \hat{n}_{i \nu \downarrow} \\
& +\sum_{i ; \nu<\nu^{\prime} ; \sigma \sigma^{\prime}}\left(V_{0}-\delta_{\sigma \sigma^{\prime}} F_{0}\right) \hat{n}_{i \nu \sigma} \hat{n}_{i \nu^{\prime} \sigma^{\prime}} \\
& -F_{0} \sum_{i ; \nu<\nu^{\prime} ; \sigma \neq \sigma^{\prime}} \hat{c}_{i \nu \sigma}^{\dagger} \hat{c}_{i \nu \sigma^{\prime}} \hat{c}_{i \nu^{\prime} \sigma^{\prime}}^{\dagger} \hat{c}_{i \nu^{\prime} \sigma}
\end{aligned}
$$

where $\nu$ denotes the two (or more) orbitals, $\sigma$ the spin, and $i, j$ the lattice sites. In this model all interactions are purely local, i.e., occur only on a single site. Apart from a Hubbard interaction $U$ for electrons of opposite spin on the same orbital, there is also a density-density interaction $V_{0}$ between electrons on different orbitals, as well as an intra-atomic exchange interaction $F_{0}$ which is separated into its density-density and spin-flip contribution (the spin-flip term is neglected in Sec. III). An "on-site pair hopping"-term of the same size is not considered since it requires an empty and a doubly-occupied orbital to take effect. Such configurations are strongly suppressed by the Hubbard interaction $U$ and the kinetic energy.
The kinetic energy describes the hopping of electrons of a given spin between identical orbitals on different sites.

It is instructive to consider the two-site model with one electron per atom, i.e., at quarter filling. In the strongcoupling regime the ground state is a spin triplet and orbital singlet, i.e., on the two atoms different orbitals are occupied. Within second order perturbation theory the energy of this state is readily calculated as $-4 t^{2} /\left(V_{0}-F_{0}\right)$. This connection between staggered orbital ordering and ferromagnetism was investigated already in 1966 by Roth [12]: applying the random phase (or Hartree-Fock) approximation to (1) she observed that, at $T=0$ and quarter filling, a ferromagnetic state is unstable against an additional staggered orbital ordering for $V_{0}-U / 2-F_{0} / 2>0$. For decreasing temperatures the Hartree-Fock approximation predicts first a phase transition from paramagnetic to ferromagnetic ordering, and at a lower temperature a second transition to a phase with ferromagnetic and orbital ordering. We note that the phenomenon of staggered orbital ordering may be a characteristic feature of any effective two-band model with orbital degeneracy (as realized, for example, in $\mathrm{e}_{\mathrm{g}}$-bands).

A major step towards the understanding of the physics of the two-band model was the derivation of an effective strongcoupling Hamiltonian at quarter filling by Kugel' and Khomskii [13, and by Cyrot and Lyon-Caen 14 who included the effect of on-site pair hopping. This effective Hamiltonian has coupled spin and orbital (pseudo spin) degrees of freedom. Within a self-consistent-field approximation 13, where orbital and spin degrees of freedom are decoupled, and also within the molecular field theory [14, where correlations between different lattice sites are neglected, the effective Hamiltonian shows an insulating ferromagnetic ground state with staggered orbital ordering. Contrary to the weak-coupling Hartree-Fock approximation this strong-coupling approach predicts that orbital ordering occurs first when the temperature is decreased 14. The ferromagnetic ground state at quarter filling and sufficiently strong Coulomb interactions was confirmed by exact diagonalization studies of finite systems in one dimension 15 17.

Off quarter or half filling the high degeneracy in the atomic limit makes a perturbational analysis essentially impossible [18. Nevertheless, for infinite Coulomb interaction $U=\infty$ and one dimension, Müller-Hartmann 2 proved that for $F_{0}>0$ the ground state of (1) is ferromagnetic for fillings $0<n<2$. Recently exact diagonalization studies in one dimension were performed by Hirsch [17]. The results depend sensitively on the boundary conditions and the number of lattice sites: below quarter filling and for six lattice sites ferromagnetism is found for the system with open boundary conditions, while it is absent when periodic boundary conditions are used. Insight was also obtained by several approximative treatments, in particular the Hartree-Fock the- 
ory [14], a generalized Hartree-Fock approach [20], more complex variational wave functions 21], the local approach [22], and the Gutzwiller approximation [23]. They all find ferromagnetism to be stabilized by Hund's rule coupling at intermediate to strong Coulomb interactions. Clearly, in this regime a proper treatment of correlation effects and the dynamics of the quantum mechanical many-body problem is essential. In the last few years the dynamical mean-field theory (DMFT) has turned out to provide a reliable framework and powerful method for the investigation of such types of problems. Hence we use it in this paper to study the stability of the ferromagnetic phase at and off quarter filling for intermediate values of the Coulomb interaction, and to determine transition temperatures.

The paper is structured as follows. In Sec. II the quantum Monte Carlo (QMC) algorithm to solve the DMFT equations is introduced. The magnetic phase diagram is presented in Sec. IIIA, and the possibility of orbital ordering is discussed in Sec. IIIB.

\section{QUANTUM MONTE CARLO SOLUTION OF THE DYNAMICAL MEAN-FIELD EQUATIONS}

The DMFT 24 30 approximates the lattice model by a single-site problem of electrons in an effective medium (meanfield) that may be described by a frequency dependent, i.e., dynamical, self-energy $\Sigma_{\nu \sigma}(\omega)$. The latter has to be determined self-consistently via a $\mathbf{k}$-integrated Dyson equation that reads

$$
G_{\nu \sigma n}=\int_{-\infty}^{\infty} d \varepsilon \frac{N^{0}(\varepsilon)}{i \omega_{n}+\mu-\Sigma_{\nu \sigma n}-\varepsilon}
$$

with Matsubara frequencies $\omega_{n}$, Green function $G_{\nu \sigma n}=$ $G_{\nu \sigma}\left(i \omega_{n}\right)$, and the DOS of the non-interacting electrons $N^{0}(\epsilon)$. In the case of the multi-band Hubbard model (1) the single-site problem takes the form

$$
G_{\nu \sigma n}=-\frac{1}{\mathcal{Z}} \int \mathcal{D}[\psi] \mathcal{D}\left[\psi^{*}\right] \psi_{\nu \sigma n} \psi_{\nu \sigma n}^{*} e^{\mathcal{A}\left[\psi, \psi^{*}, \mathcal{G}^{-1}\right]}
$$

where $\psi$ and $\psi^{*}$ are Grassmann variables, $\mathcal{G}^{-1}=G^{-1}+\Sigma$, and $\mathcal{A}\left[\psi, \psi^{*}, \mathcal{G}^{-1}\right]$ denotes the single-site action including all local interactions

$$
\begin{aligned}
& \mathcal{A}\left[\psi, \psi^{*}, \mathcal{G}^{-1}\right]=\sum_{\nu \sigma, n} \psi_{\nu \sigma n}^{*} \mathcal{G}_{\nu \sigma n}^{-1} \psi_{\nu \sigma n} \\
& -U \sum_{\nu} \int_{0}^{\beta} d \tau \psi_{\nu \uparrow}^{*}(\tau) \psi_{\nu \uparrow}(\tau) \psi_{\nu \downarrow}^{*}(\tau) \psi_{\nu \downarrow}(\tau) \\
& -\sum_{\nu<\nu^{\prime} ; \sigma \sigma^{\prime}}\left(V_{0}-\delta_{\sigma \sigma^{\prime}} F_{0}\right) \int_{0}^{\beta} d \tau \psi_{\nu \sigma}^{*}(\tau) \psi_{\nu \sigma}(\tau) \psi_{\nu^{\prime} \sigma^{\prime}}^{*}(\tau) \psi_{\nu^{\prime} \sigma^{\prime}}(\tau) \\
& +F_{0} \sum_{\nu<\nu^{\prime} ; \sigma \neq \sigma^{\prime}} \int_{0}^{\beta} d \tau \psi_{\nu \sigma}^{*}(\tau) \psi_{\nu \sigma^{\prime}}(\tau) \psi_{\nu^{\prime} \sigma^{\prime}}^{*}(\tau) \psi_{\nu^{\prime} \sigma}(\tau) .
\end{aligned}
$$

As for the one-band model [27,28] Eqn. (3) is equivalent to a (degenerate) Anderson impurity model and can thus be treated by standard techniques. In the present paper the QMC algorithm of Hirsch and Fye 31 will be used. In a first step each one of the four terms of Eqn. (4) are decomposed via the Trotter-Suzuki formula, and imaginary time is discretized $(\tau=l \Delta \tau$ for $l=1 \ldots \Lambda$ and $\Delta \tau=\beta / \Lambda)$. In the second step the interaction terms are decoupled to obtain a quadratic action. For the density-density interactions this is achieved as usual by the Hubbard-Stratonovich transformation

$$
\begin{array}{r}
\exp \left\{\frac{\Delta \tau}{2}\left(V_{0}-F_{0}\right)\left(\psi_{\nu \sigma l}^{*} \psi_{\nu \sigma l}-\psi_{\nu^{\prime} \sigma l}^{*} \psi_{\nu^{\prime} \sigma l}\right)^{2}\right\}= \\
\frac{1}{2} \sum_{s= \pm 1} \exp \left\{\lambda s\left(\psi_{\nu \sigma l}^{*} \psi_{\nu \sigma l}-\psi_{\nu^{\prime} \sigma l}^{*} \psi_{\nu^{\prime} \sigma l}\right)\right\}
\end{array}
$$

where $\cosh (\lambda)=\exp \left(\Delta \tau\left(V_{0}-F_{0}\right) / 2\right)$. The same decoupling holds for the remaining $V_{0}$ and the $U$ terms. For $N$ orbitals this yields altogether $\left(2 N^{2}-N\right) \Lambda$ auxiliary fields for the density-density interactions.

By contrast, there does not exist a standard decoupling scheme for the last term of Eqn. (4) describing a spin-flip. Recently Motome and Imada 32 proposed a decoupling scheme for this term: it avoids the minus-sign problem in the symmetric case at half filling, but leads to a phase problem off half filling due to the use of a complex auxiliary field. Instead, we tried the following Hubbard-Stratonovich decoupling:

$$
\begin{aligned}
& \exp \left(\Delta \tau F_{0} \psi_{\nu \sigma l}^{*} \psi_{\nu-\sigma l} \psi_{\nu^{\prime}-\sigma l}^{*} \psi_{\nu^{\prime} \sigma l}\right)= \\
& \frac{1}{2} \sum_{s= \pm 1} \exp \left\{\mu s\left(\psi_{\nu \sigma l}^{*} \psi_{\nu-\sigma l}+\psi_{\nu^{\prime}-\sigma l}^{*} \psi_{\nu^{\prime} \sigma l}\right)\right\}
\end{aligned}
$$

with $\mu=\sqrt{\Delta \tau F_{0}}$. Unfortunately, this transformation was found to lead to a sign-problem, too, even within the DMFT where the sign-problem is absent in the single-band case. For this reason we neglect the spin-flip term in the following. Hund's rule coupling is thus restricted to the direction of the quantization axis, i.e., the $z$-axis, implying the breaking of the $\mathrm{SU}(2)$ spin rotation symmetry. The restriction to an Ising-type Hund's rule coupling is commonly used in the investigations of the two-band Hubbard model [12, 14, 19, 20]. This restriction has no effect on the critical temperatures in the limits of weak and strong coupling, i.e., in the HartreeFock approximation and the Weiss mean-field theory of the corresponding spin model, respectively. Therefore we expect that the spin-flip term has no strong effect on the critical temperature at intermediate coupling. Note, that while the influence of the spin-flip term on critical temperatures is probably small the excitation spectrum at $\mathrm{T}=0$ depends sensitively on this term. It shows a spin gap or not, respectively.

Based on the above Hubbard-Stratonovich decoupling the Monte Carlo method is employed to sample the auxiliary spin configurations and thus to calculate Green functions and susceptibilities. The multi-band algorithm is a generalization of the one-band algorithm. A similar generalization was employed by Rozenberg [33] in the investigation of metalinsulator transitions in the two-band Hubbard model without Hund's rule coupling.

Phase boundaries are determined by a Curie-Weiss fit of the corresponding susceptibility in the homogeneous phase. Since the corresponding critical temperatures still depend on the unphysical time discretization parameter $\Delta \tau$ a second order fit to $\Delta \tau=0$ was performed from at least six values of 
$\Delta \tau \in[0.075,0.5]$. Besides the statistical error of the QMC simulation (propagated via $T$ - and $\Delta \tau$-fit), there exists an additional systematic error due to higher order contributions in the $T$ and $\Delta \tau$ fits. In particular the $\Delta \tau$ dependence of the Curie temperature was considerable for the data of Sec. III. Therefore we estimated this systematic error by comparing the Curie temperature obtained from all $\Delta \tau$ values to that calculated without the $\Delta \tau=0.5$ value. The individual and mean difference between these two fits was within the statistical error of the Monte Carlo data. This analysis implies that the systematic error is smaller than the statistical error shown in the figures.

\section{RESULTS AND DISCUSSION}

\section{A. Ferromagnetism}

In the present paper we investigate the Hund's rule coupling in the presence of orbital degeneracy as a possible origin of itinerant ferromagnetism. This microscopic mechanism should be distinguished from the one found to be important for the single-band Hubbard model, which is based on an asymmetric DOS [1 [9]. Therefore we employ a symmetric semielliptic DOS $N^{0}(\epsilon)=\sqrt{\left(2 t^{*}\right)^{2}-\epsilon^{2}} /\left(2 \pi t^{* 2}\right)$ (in the following $t^{*} \equiv 1$ will set our energy scale). For this DOS and a symmetric Gaussian DOS no ferromagnetism was found in the single-band Hubbard model up to a Coulomb interaction of $U=30$ [8, 9 .

In fact, even the two-band model without Hund's rule coupling does not indicate ferromagnetism at $U=9, V_{0}=5$, and $n=1.25$ (see Fig. 11). However, already a small Hund's rule coupling, $F_{0}=1.61 \pm 0.15$, is sufficient to stabilize ferromagnetism [34]. This value is considerably smaller than that obtained by Kuei and Scalettar [16]: in one dimension (six sites) at $n=1$ with $U=V_{0}+F_{0}$ no ferromagnetism was found below $F_{0} \approx 8$ (in units rescaled to obtain a bandwidth of four). This value of $F_{0}$ is, however, comparable to the

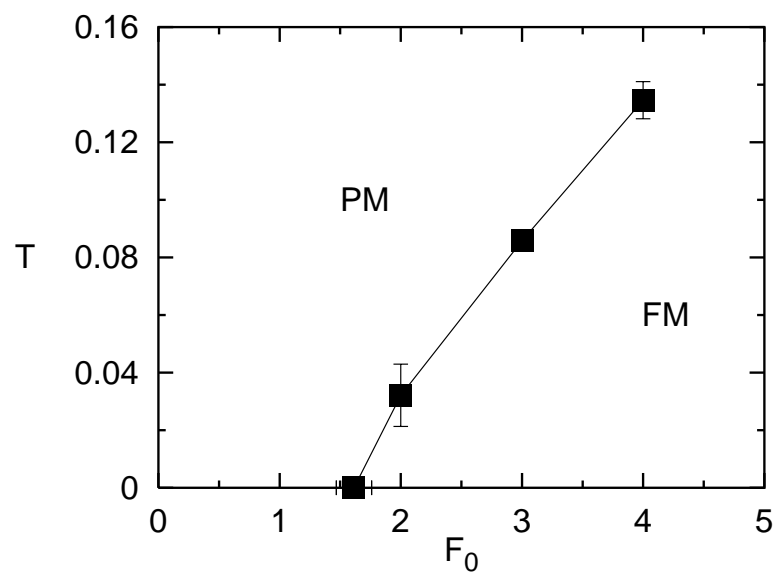

FIG. 1. Curie temperature vs. Hund's rule coupling $F_{0}$ for the two-band Hubbard model at $U=9, V_{0}=5$, and $n=1.25$. Ferromagnetism is seen to be stabilized by Hund's rule coupling.

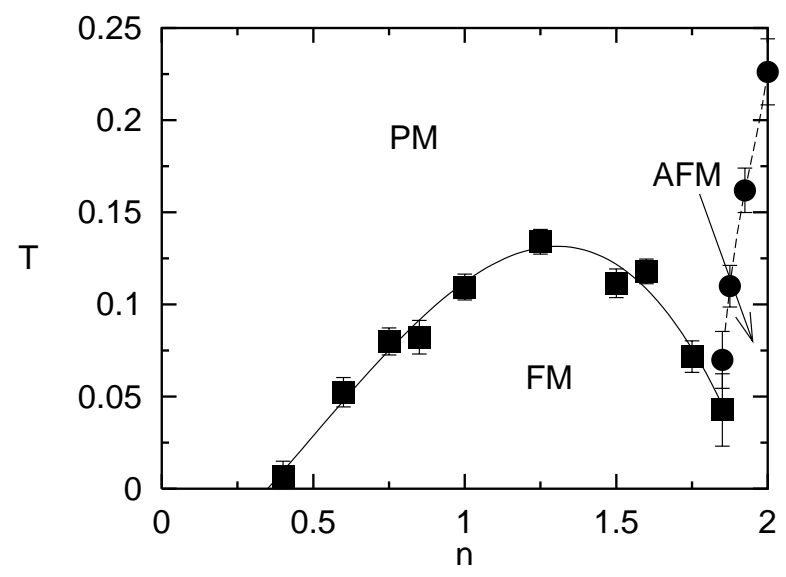

FIG. 2. Magnetic $T-n$ phase diagram for $U=9$, $V_{0}=5$, and $F_{0}=4$ with paramagnetic (PM), ferromagnetic $(\mathrm{FM})$, and antiferromagnetic phase (AFM). Ferromagnetism is found in a broad range of fillings.

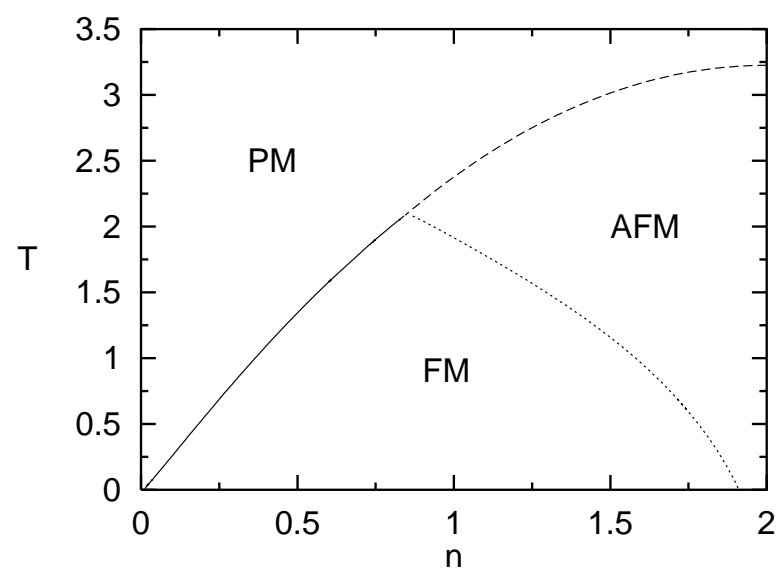

FIG. 3. Same as Fig. 2 but calculated within the Hartree-Fock approximation (solid line: Curie temperature, dashed line: Néel temperature, dotted line: first order transition between FM and AFM).

results of Hirsch 177: in one dimension at $n=1$ and $n=$ 1.5 (six and four sites, respectively) with $U=V_{0}+2 F_{0}$ no ferromagnetism was found below $F_{0} \approx 1.5$ and 2 , respectively. Note, that the relation $U=V_{0}+F_{0}$ makes the Hamiltonian (11) form-invariant w.r.t. orbital rotations, while this holds for $U=V_{0}+2 F_{0}$ if an additional pair hopping term is added. The Hund's rule coupling $F_{0}$ must be smaller than the densitydensity interaction $V_{0}$ since otherwise an unphysical attractive interaction between two electrons on the same site (in different orbitals with the same spin) exists.

The magnetic $T-n$ phase diagram is presented in Fig. 2 for $U=9, V_{0}=5$, and a relatively strong Hund's rule coupling $F_{0}=4$. Ferromagnetism is found in a broad range of electron densities $n$. Note, that the phase-diagram is symmetric around $n=2$ due to particle-hole symmetry. The special case of quarter filling $n=1$ does not mark a pronounced point in the phase diagram. In particular the maximal Curie temperature is found above quarter filling. Typical Curie tempera- 
tures are about 0.1 , which for a bandwidth of $4 \mathrm{eV}\left(t^{*} \equiv 1 \mathrm{eV}\right)$ corresponds to $0.1 \mathrm{eV}$, i.e., about $1000 \mathrm{~K}$. Near half filling the antiferromagnetic Heisenberg exchange suppresses the ferromagnetic order and a narrow antiferromagnetic phase with the usual AB sublattice structure develops. Fig. 1 suggests that Curie and Néel temperature cross at a finite temperature and not at $T=0$. This is confirmed by the observation that for $n=1.85$ the Curie temperature extrapolated from the paramagnetic phase lies only slightly below the Néel temperature.

The same $T-n$ phase diagram calculated within the Hartree-Fock approximation is shown in Fig. 3. The HartreeFock approximation fails to describe the suppression of the magnetic order at the crossover from ferro- to antiferromagnetism. Furthermore, the magnetic phases are overestimated, i.e., the critical temperatures are more than an order of magnitude too large and both magnetic phases continue to extremely small values of $n$. Within the Hartree-Fock approximation a first order phase transition between antiferromagnetic and ferromagnetic phase occurs. It is not clear at present whether this is also true within the DMFT.

\section{B. Orbital ordering}

As was pointed out in the introduction, at quarter filling the Weiss mean-field theory for the effective Kugel' and Khomskii Hamiltonian [13, 14] predicts an instability of the paramagnetic phase against staggered orbital ordering when the temperature is decreased, while a transition to pure ferromagnetic order is suggested by the Hartree-Fock approximation. The DMFT, containing both approximations as limits at strong and weak coupling, respectively, is well suited to clarify this contradiction. To investigate a possible transition from the phase without long-range order to a phase with mixed ferromagnetic and staggered orbital ordering we introduce a parameter $\delta$ that allows one to investigate the instability w.r.t. a general mixed ordering. For every $\delta$ the corresponding susceptibility is defined through a field $h$, which modifies the grand potential $\Omega$ by the term

$$
\begin{aligned}
\hat{H}_{h} & =-h \sum_{i \nu \sigma} \hat{n}_{i \nu \sigma}\left\{(1-\delta) \sigma+\delta(-1)^{i} \nu\right\} \\
\chi & =-\frac{1}{L} \frac{\partial^{2} \Omega}{\partial h^{2}} .
\end{aligned}
$$

Here $L$ denotes the number of lattice sites. For $\delta=0$ one obtains the (para-) magnetic susceptibility and for $\delta=1$ the susceptibility for orbital ordering. Fig. 4 shows that for $U=9$, $V_{0}=5$, and $F_{0}=4$ the critical temperature is maximal for $\delta=0$, i.e., the paramagnet is unstable against pure ferromagnetic order. Thus, the phase diagram Fig. 1 need not be modified by phase transitions from paramagnetic to orbital ordering. Whether the ferromagnetic phase becomes unstable against orbital ordering at even lower temperatures cannot be answered by the method employed here, i.e., by the calculation of susceptibilities within the paramagnetic phase.

The scenario of Fig. 4 qualitatively agrees with the HartreeFock approximation, which even at $T=0$ does not predict orbital ordering since $V_{0}-U / 2-F_{0} / 2<0$. The disagreement with Weiss mean-field theory can be explained by the fact

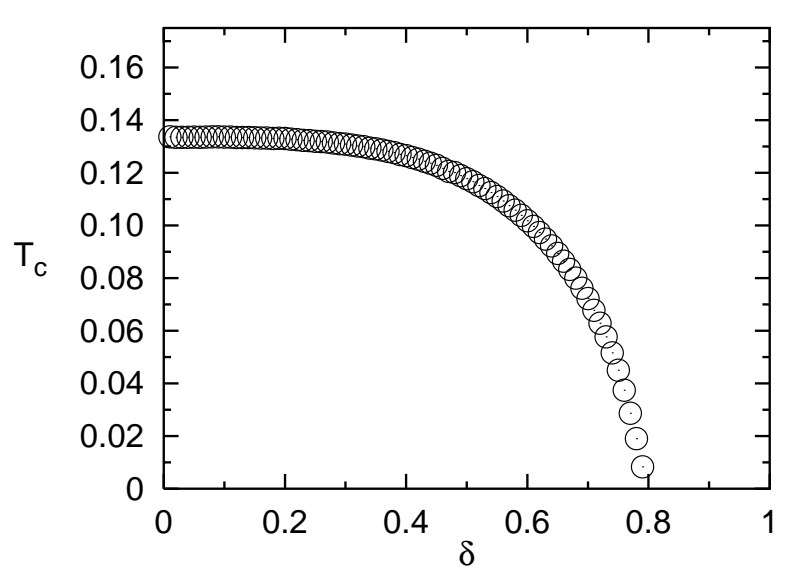

FIG. 4. Critical temperature for the instability against a state with mixed ferromagnetic and orbital ordering (control parameter $\delta$ ) as obtained from $\left[\chi\left(T_{c}, \delta\right)\right]^{-1}=0$. For $U=9$, $V_{0}=5, F_{0}=4$, and $n=1$ the highest temperature is found at $\delta=0$, indicating a transition into a purely ferromagnetic state.

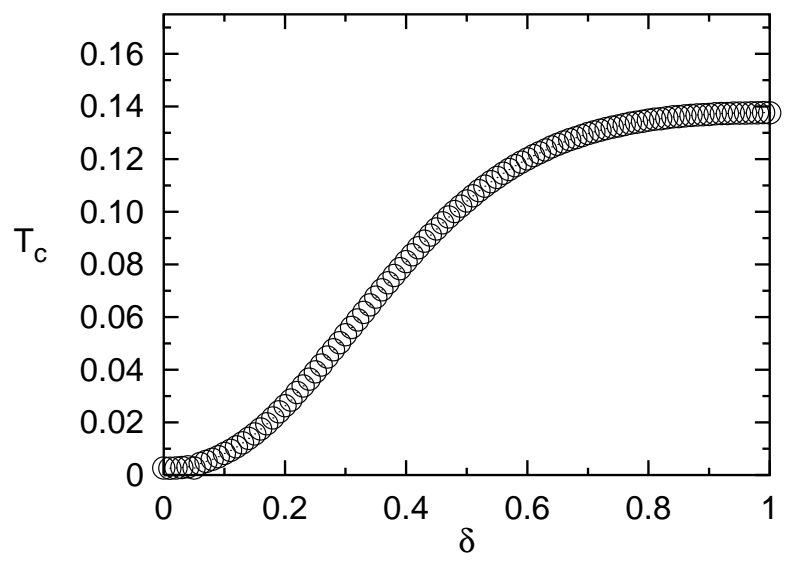

FIG. 5. Same as in Fig. 4 but for $U=8, V_{0}=6, F_{0}=2$, and $n=1$. Here, the maximal temperature is found at $\delta=1$, i.e., an instability against staggered orbital ordering occurs.

that the second order perturbation theory leading to the effective strong-coupling Hamiltonian is controlled in $t^{2} /\left(V_{0}-\right.$ $\left.F_{0}\right)$ which is of $\mathcal{O}(1)$ here.

The considerations above suggest that orbital ordering may occur if the intra-atomic exchange $F_{0}$ becomes smaller. Indeed, for $U=8, V_{0}=6$, and $F_{0}=2$ a phase transition to pure orbital ordering $(\delta=1)$ occurs (see Fig. 5). Fig. 6] shows the $T-n$ phase diagram for these parameters, including orbital ordering near quarter filling, antiferromagnetism near half filling and ferromagnetism in between. We should mention, that although at $n=0.9$ the inverse orbital ordering susceptibility decreases with decreasing temperatures in a Curie-Weiss like behavior, suggesting a transition at $T \approx 0.04$, it decreases again at lower temperatures. Hence orbital ordering does not take place at $n=0.9$.

Even at $n=1.2$, i.e., the crossing point of the orbital ordering and ferromagnetic phase boundary in Fig. 6, no instability 
against a mixed ferromagnetic and orbital ordering is found. Therefore we conclude that the two-band Hubbard model with Hund's rule coupling $F_{0}$ shows an instability towards either pure ferromagnetic or pure orbital ordering. However, since these phases do not exclude each other a phase with mixed order may appear at even lower temperatures, as is predicted by Hartree-Fock and Weiss mean-field theory.

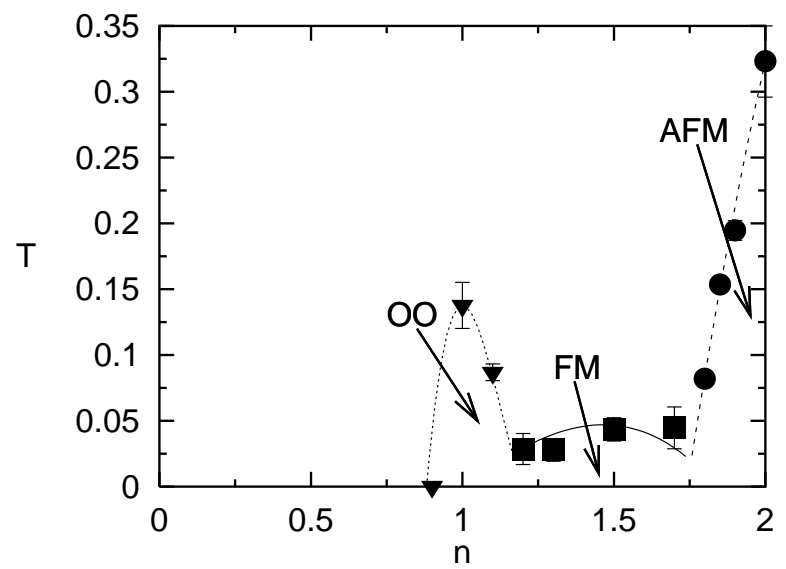

FIG. 6. $T-n$ phase diagram for $U=8, V_{0}=6$, and $F_{0}=2$. In addition to the phases in Fig. 2 an orbital ordering (OO) phase is found near quarter filling.

\section{Conclusion}

We showed that even for a symmetric DOS the Hund's rule coupling provides an effective microscopic mechanism for the stabilization of ferromagnetism in a broad range of electron densities off half filling. This mechanism takes effect at intermediate to strong values of the Coulomb interaction and therefore requires a proper treatment of the quantum mechanical correlations. It is different from the mechanism based on an asymmetric DOS, which leads to ferromagnetism even in the single-band Hubbard model. The question which one of these mechanisms is the main driving force for ferromagnetism in $\mathrm{Fe}, \mathrm{Co}$, and $\mathrm{Ni}$ remains open, if it can be explained by a single mechanism at all.

\section{Acknowledgments}

The authors acknowledge helpful discussions with M. Ulmke, J. Schlipf, and M. Kollar. A generous computing account on the VPP 700 was provided by the LRZ München.

[1] A. Mielke and H. Tasaki, Commun. Math. Phys. 158, 341 (1993); H. Tasaki, preprint cond-mat/9712219 (1997).

[2] E. Müller-Hartmann, J. Low Temp. Phys. 99, 349 (1995).

[3] T. Hanisch, G. S. Uhrig, and E. Müller-Hartmann, Phys. Rev. B 56, 13960 (1997).
[4] S. Daul and R. Noack, Z. Phys. B 103, 293 (1997).

[5] R. Hlubina, S. Sorella, and F. Guinea, Phys. Rev. Lett. 78, 1343 (1997).

[6] D. Vollhardt, N. Blümer, K. Held, M. Kollar, J. Schlipf, and M. Ulmke, Z. Phys. B 103, 283 (1997).

[7] M. Ulmke, Euro. Phys. J. B (in press).

[8] J. Wahle, N. Blümer, J. Schlipf, K. Held, and D. Vollhardt, preprint cond-mat/9711242 (1997).

[9] Itinerant ferromagnetism was found to be stable even for a symmetric DOS at very large values of the Coulomb interaction, see T. Obermeier, T. Pruschke, and J. Keller, Phys. Rev. B 56, R8479 (1997).

[10] J. C. Slater, Phys. Rev. 49, 537 (1936).

[11] J. H. van Vleck, Rev. Mod. Phys. 25, 220 (1953).

[12] L. M. Roth, Phys. Rev. 149, 306 (1966).

[13] K. I. Kugel' and D. I. Khomskii, Sov. Phys.-JETP 37, 725 (1973).

[14] M. Cyrot and C. Lyon-Caen, J. Phys. C 36, 253 (1975).

[15] W. Gill and D. J. Scalapino, Phys. Rev. B 35, 215 (1987).

[16] J. Kuei and R. T. Scalettar, Phys. Rev. B 55, 14968 (1997).

[17] J. E. Hirsch, Phys. Rev. B 56, 11022 (1997).

[18] In the strong coupling limit a canonical transformation was employed by K. A. Chao, J. Spałek, and A. M. Oleś, Phys. Stat. Sol. B 84747 (1977) to obtain an effective Hamiltonian at arbitrary fillings. The ground state of this complicated effective Hamiltonian was analyzed at quarter and half-filling.

[19] A. M. Oleś, Phys. Rev. B 28, 327 (1983).

[20] M. Fleck, A. M. Oleś, and L. Hedin, Phys. Rev. B 56, 3159 (1997).

[21] T. Okabe, Prog. Theor. Phys. 98, 331 (1997).

[22] A. M. Oleś, Phys. Rev. B 23, 271 (1981); A. M. Oleś and G. Stollhoff Phys. Rev. B 29, 314 (1984).

[23] J. Bünemann and W. Weber, Physica B 230, 412 (1997); J. Bünemann, F. Gebhard, and W. Weber, J. Phys. Condens. Matter 9, 7343 (1997); J. Bünemann, W. Weber, and F. Gebhard, to be published.

[24] W. Metzner and D. Vollhardt, Phys. Rev. Lett. 62, 324 (1989).

[25] E. Müller-Hartmann, Z. Phys. B 74, 507 (1989).

[26] V. Janiš, Z. Phys. B 83, 227 (1991); V. Janiš and D. Vollhardt, Int. J. Mod. Phys. B 6, 731 (1992).

[27] M. Jarrell, Phys. Rev. Lett. 69, 168 (1992).

[28] A. Georges and G. Kotliar, Phys. Rev. B 45, 6479 (1992).

[29] D. Vollhardt in Correlated Electron Systems, edited by V. J. Emery (World Scientific, Singapore, 1993), p.57.

[30] A. Georges, G. Kotliar, W. Krauth, and M. Rozenberg, Rev. Mod. Phys. 68, 13 (1996).

[31] J. E. Hirsch and R. M. Fye, Phys. Rev. Lett. 56, 2521 (1986).

[32] Y. Motome and M. Imada, J. Phys. Soc. Jpn. 66, 1872 (1997).

[33] M. J. Rozenberg, Phys. Rev. B 55, R4855 (1997).

[34] The critical value of $F_{0}$ was determined by a linear interpolation between the Curie temperature for $F_{0}=2$ and the (negative) Curie temperature for $F_{0}=1$. 